\title{
Déterminisme des Coassements des Anoures de la ferme piscicole du Parc National du Banco (Côte d'Ivoire)
}

\author{
Blayda TOHE ${ }^{1 *}$, N'Guessan E. AssemiAn ${ }^{1}$, N'Goran G. KouAme ${ }^{1}$, Germain GourenE ${ }^{1}$ \& Mark-Oliver RöDEL ${ }^{2}$ \\ ${ }^{1}$ Université d'Abobo-Adjamé, Laboratoire d'Environnement et de Biologie Aquatique, UFR-SGE, 02 B.P. 801 Abidjan 02 \\ (Côte d'lvoire) \\ ${ }^{2}$ Theodor-Boveri-Institute, Department of Animal Ecology and Tropical Biology (Zoology III), Am Hubland, D-97074 Wûrzburg, Germany. \\ *Auteur pour les correspondances (E-mail : toheblayda@yahoo.fr) \\ Reçu le 20-10-2006, accepté le 14-02-2008.
}

\section{Résumé}

Les facteurs déterminant les activités de coassements des anoures de la ferme piscicole du Parc National du Banco ont été étudiés. Une prospection acoustique basée sur l'enregistrement standardisé des chants des grenouilles, a permis d'inventorier, pendant quatre cycles nycthéméraux, 14 espèces réparties en 8 genres et 4 familles. La famille des Hyperoliidae avec 7 espèces est la plus diversifiée. Parmi les espèces recensées, une seule (Phrynobatrachus accraensis), a son pic d'activité à 6 heures. Son rythme d'activité de chants est influencé par la température de l'air et l'intensité lumineuse. Les Bufonidae, Hyperoliidae et Ranidae constituent une communauté aux mœurs nocturnes dont les pics de coassements se situent entre 21 heures et 24 heures. L'humidité relative de l'air conditionne leurs comportements. Par ailleurs, la majorité des espèces a une forte activité de coassement en saison de pluies. Nos résultats qui sont parmi les premiers concernant les communautés d'Anoures du Parc National du Banco, permettront d'élaborer une stratégie pour leur échantillonnage et leur protection.

Mots clés : Coassements, Variations Temporelles, Amphibiens, Parc National du Banco, Afrique de l'Ouest.

\begin{abstract}
West African Anura Calling Activities (Banco National Park fish farm - Côte d'lvoire)

The factors determining the calling activities of anuran community in the Banco National Park fish farm have been studied. The standard acoustic encounter survey based on listening calling males permitted to inventory 14 species, 8 genera and 4 families, during four circadian cycles. The family of Hyperoliidae is diversified the most (7 species). Only one diurnal species (Phrynobatrachus accraensis) was observed, with a peak of activity at 6 hours. Its daily calling activity is influenced by the temperature of the air and the light intensity. The Bufonidae, Hyperoliidae and Ranidae families are nocturnal community. Their peaks of activity varied between 21 hours and 24 hours. The relative humidity of the air determined the behavior of these families. In addition, most of the species have a strong calling activity during the rainy season. Our results should permit to elaborate a standard method for amphibians survey in the Park.
\end{abstract}

Key words : Calling activity, Temporal variation, Amphibian, Banco National Park, West Africa.

\section{Introduction}

Les populations d'Amphibiens sont en plein déclin à travers le monde (Wake, 1991 ; Blaustein
\& Kiesecker, 2002). Cette situation est due à l'action combinée des changements climatiques, des pollutions chimiques, des maladies infectieuses, de la dégradation des habitats par 
les activités agricoles et autres phénomènes liés au développement (Kiesecker \& Blaustein, 1995 ; Hilton-Taylor, 2000 ; Kiesecker et al., 2001 ; Williams et al., 2002 ; Blaustein et al., 2003 ; Daszak et al., 2003). De même, l'utilisation des grenouilles comme animaux de laboratoire et nourriture constituent également de graves menaces (Patel, 1993 ; Benito-Espinal, 1997 ; Veith et al., 2000 ; Channing, 2001).

Aussi, la récente prise de conscience relative à la disparition massive de ces animaux a-t-elle permis de promouvoir des techniques d'échantillonnage écologiques fiables (Kiesecker \& Blaustein, 1998 ; Biek et al., 2002). Elles sont basées sur l'enregistrement standardisé des coassements (Heyer et al., 1994) et constituent une approche adéquate pour le suivi des populations d'Anoures (Rödel \& Ernst, 2004).

Les coassements ou chants des grenouilles sont surtout liés à la reproduction (Wells 1977 ; Heyer et al., 1994). Ce sont les mâles qui utilisent ces chants comme des appels sexuels. Mais ils peuvent aussi traduire des modifications des conditions du milieu (Scott, 1976; Toft, 1980; Lamotte \& Sanchez-Lamotte,1999 et Ovaska \& Rand, 2001). De nombreux travaux relatifs aux variations journalières des coassements ont été réalisés en Europe, Amérique et Asie (Given, 1987 ; Shimoyama, 1989 ; Runckle et al., 1994 ; Bevier, 1997 ; Bridges \& Dorcas, 2000). En Afrique, notamment en Côte d'Ivoire, la plupart des travaux sont assez récents et ne concernent que deux Arthroleptidae du Parc National de Taï (Leistner, 2001).

La présente étude a pour objectif de déterminer le rythme d'activité journalier de chants des grenouilles de la ferme piscicole du Parc National du Banco (PNB). Elle vise également à mettre en évidence les facteurs du milieu qui l'influencent. Ce site a été choisi pour sa forte richesse spécifique (Assemian et al., 2006).

\section{Matériel et méthodes}

\subsection{Milieu d'étude}

Le Parc National du Banco $\left(5^{\circ} 21^{\prime}-5^{\circ} 25^{\prime} \mathrm{N}\right.$ et $\left.4^{\circ} 01^{\prime}-4^{\circ} 05^{\prime} \mathrm{W}\right)$, relique de forêt primaire psammohygrophile de plus de 3000 ha (Daget et Iltis, 1965) est situé à Abidjan, la capitale économique de la Côte d'Ivoire (Fig. 1). Cette forêt ombrophile est soumise à un climat tropical à quatre saisons ; deux saisons sèches (août à septembre et décembre à mars) et deux saisons pluvieuses (avril à juillet et octobre à novembre). Elle est caractérisée par une diversité de microhabitats naturels et anthropiques, dont une ferme piscicole d'une superficie de 2,5 ha située entre $5^{\circ} 23^{\prime} 06^{\prime \prime} \mathrm{N}$ et $4^{\circ} 03^{\prime} 06^{\prime \prime} \mathrm{W}$. Ce site qui avait été aménagé pour servir de laboratoire à l'Ecole Forestière du Banco, abrite 16 étangs qui sont curés deux fois par an. La végétation de cette ferme est composée d'importantes formations herbeuses (Graminées) et de fougères au Sud, de bambous à l'Est et à l'Ouest alors qu'à la lisière Nord, se trouve une forêt marécageuse. La ferme est irriguée en permanence par la rivière Banco et est sujette à un désherbage et brûlis trimestriels.

\subsection{Colette des données}

La méthode d'échantillonnage acoustique décrite par Zimmerman (1994) a été utilisée pour notre étude. Cette technique est peu onéreuse en temps et en matériel (Crump \& Scott, 1994 ; Zimmerman, 1994). Elle consiste à identifier les coassements et à dénombrer les individus qui chantent.

Quatre cycles de 24 heures, deux en saison sèche (septembre 2004 et janvier 2005) et deux en saison pluvieuse (mai 2004 et juin 2004) ont été réalisés dans la ferme piscicole du Banco. Les données climatiques (intensité lumineuse, température, humidité relative) ont été relevées à l'aide d'un multimètre Conrad version 02/02 en même temps que le comptage des chants au cours d'un cycle de 24 heures. Les enregistrements de coassements ont été effectués toutes les 3 heures (3h ; 6h ; 9h ; 12h ; 15h ; 18h ; 21h ; 24h) pendant 15 minutes soit 120 minutes. L'identification (taxinomique) des chants des grenouilles s'est faite grâce à un répertoire audio contenu sur un CDRom (Rödel, 2000). Ce répertoire renferme les coassements de 24 espèces d'Anoures africains.

\subsection{Analyse des données}

Afin de comparer les variations journalières et saisonnières de l'humidité relative, la température de l'air et l'intensité lumineuse, nous avons utilisé le test non paramétrique de MannWithney à l'aide du logiciel STATISTICA version 7.1. Ce logiciel a également permis de calculer les coefficients de corrélation entre les paramètres climatiques et les abondances des chants des grenouilles. 
La méthode RDA (ReDundancy Analysis) qui met en relation les trois paramètres environnementaux, les espèces d'amphibiens et leurs variations journalière et saisonnière a été réalisée à l'aide du logiciel CANOCO (Cononical Community Ordination, version 4.5).

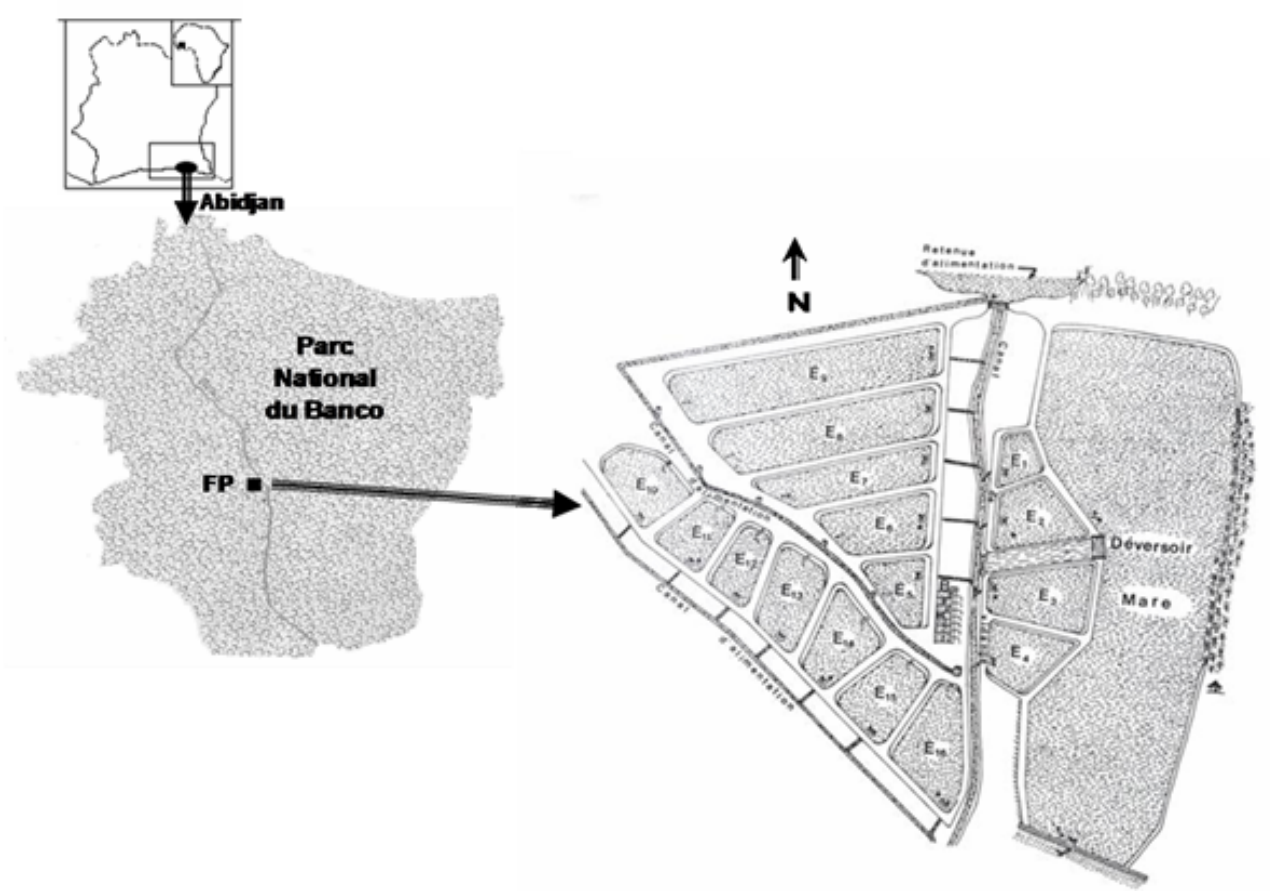

Figure 1 : Situation géographique du site d'échantillonnage dans le Parc National du Banco. $F P=$ Ferme Piscicole $; E 1$ à E16 = étangs ou bassins piscicoles.

\section{Résultats}

\subsection{Données climatiques}

II n'y a pas de différences significatives entre les données saisonnières et journalières $(0,083=P$ $=1$ ) des quatre rythmes à l'exception de l'intensité lumineuse dont les valeurs diurnes sont significativement différentes des valeurs nocturnes $(P<0,01)$ (Fig. 2).

Les températures moyennes journalières les plus élevées ont été enregistrées en janvier et mai (27 ${ }^{\circ} \mathrm{C}$ ) et les plus faibles en juin $\left(23,5^{\circ} \mathrm{C}\right)$ et septembre $\left(24^{\circ} \mathrm{C}\right)$. Cependant, les plus importantes variations journalières ont été observées en janvier et mai avec les valeurs extrêmes comprises entre 23 et $42^{\circ} \mathrm{C}$. Les plus faibles variations ont été obtenues en septembre (23 à $\left.29,5^{\circ} \mathrm{C}\right)$.

L'humidité relative de l'air est plus élevée en juin (92\%) et faible en janvier (83\%). Les écarts les plus importants ont été enregistrés en mai (50 à $95 \%)$. L'air est plus humide dans le mois de septembre (70 à $95 \%$ ).

Concernant l'intensité lumineuse, les valeurs les plus importantes ont été enregistrées en janvier (3500 lux) avec les extrêmes comprises entre 27 et 9790 lux. Septembre est le mois le moins éclairé avec $75 \%$ des intensités lumineuses en dessous de 566 lux.

\subsection{Richesse spécifique}

La ferme piscicole du Banco est riche de 14 espèces d'Anoures reparties en 8 genres et 4 familles (Tableau 1). Cette communauté est dominée par les grenouilles arboricoles (50 $\%$ ) et les grenouilles des litières (42,9\%). La famille des Hyperoliidae est la plus diversifiée avec 7 espèces (50\%) reparties entre trois genres. Les Petropedetidae constituent la famille la moins représentée avec un seul genre et une espèce $(7,1 \%)$. 

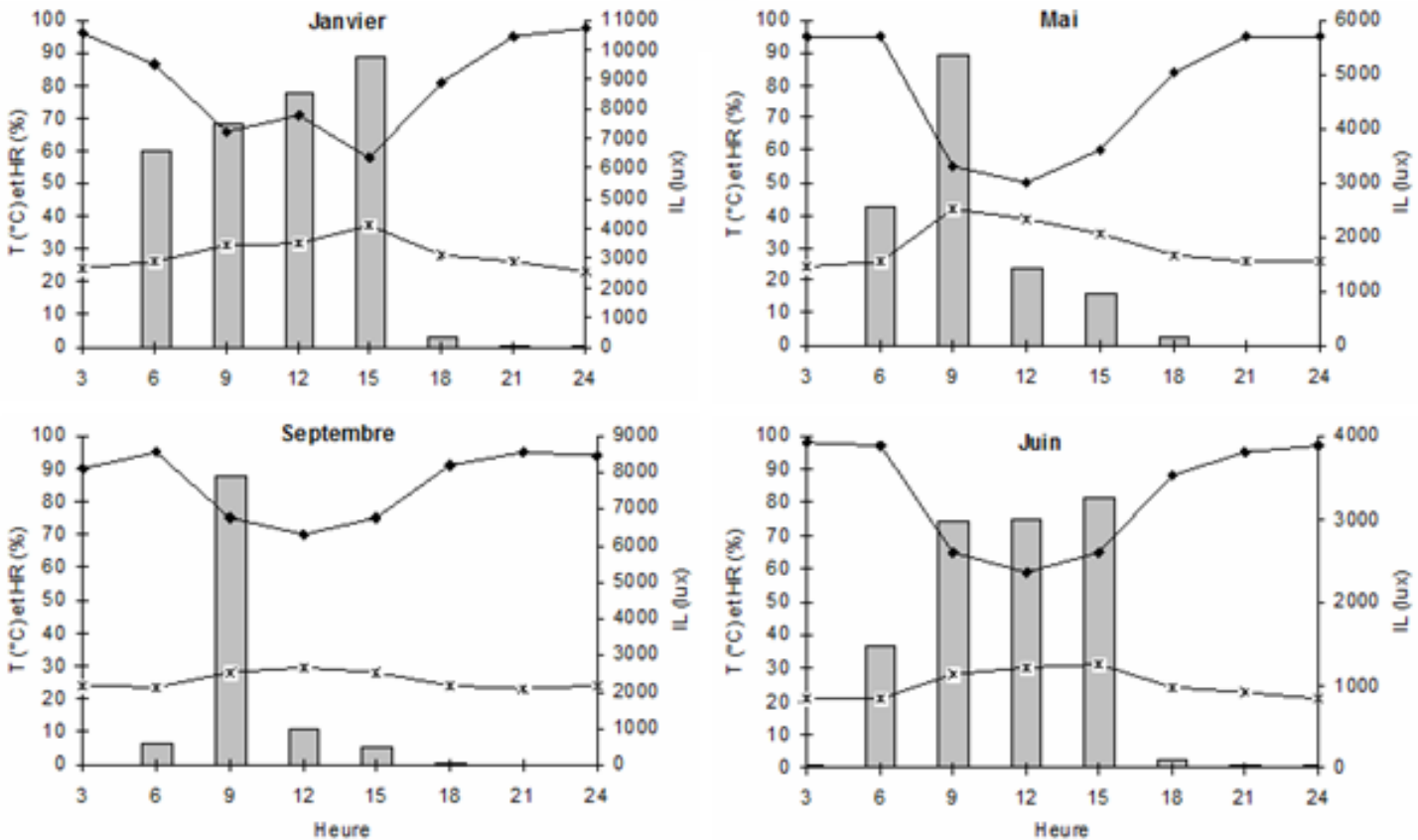

๑IL (lux)

$-\mathrm{x}-\mathrm{T}(\mathrm{C})$

$\rightarrow H R(\%)$

Figure 2: Variation journalière de l'intensité lumineuse (IL), de la température (T) et de l'humidité relative de l'air (HR) de la ferme piscicole du Parc National du Banco pendant les saisons sèche (janvier et septembre) et pluvieuse (mai et juin)

Tableau 1: Composition spécifique des Amphibiens de la ferme piscicole du Parc National du Banco et leurs habitats préférés.

\begin{tabular}{lcl}
\hline Taxons & Fréquences (\%) & Habitats préférés \\
\hline Bufonidae & 14,3 & \\
\hline Bufo maculatus & & litière \\
Bufo regularis & & litière \\
\hline Hyperoliidae & 50 & \\
\hline Afrixalus dorsalis & arboricole \\
Hyperolius guttulatus & & arboricole \\
Hyperolius fusciventris & & arboricole \\
Hyperolius picturatus & & arboricole \\
Hyperolius concolor & arboricole \\
Hyperoliidae nov. gen. nov. sp. & & arboricole \\
Leptopelis hyloides & arboricole \\
\hline Petropedetidae & \\
\hline Phrynobatrachus accraensis & 7,1 & \\
\hline Ranidae & litière \\
\hline Amnirana albolabris & \\
Hoplobatrachus occipitalis & litière \\
Ptychadena mascareniensis & aquatique \\
Ptychadena pumilio & litière \\
\hline & & litière \\
\hline
\end{tabular}




\subsection{Variation du rythme journalier des coassements en fonction des saisons}

Un total de 2734 chants ont été dénombrés à la ferme piscicole. Cet effectif se repartit comme suit par ordre décroissant d'importance : Phrynobatrachus accraensis (666 chants, soit 24,4 $\%$ ), Hyperolius guttulatus (456 chants, soit 16,7 $\%)$, Afrixalus dorsalis (442 chants, soit 16,2\%), Ptychadena pumilio (276 chants, soit 10,1\%), Hyperolius fusciventris (262 chants, soit 9,6\%), Ptychadena mascareniensis (205 chants, soit 7,5 $\%$ ), Hoplobatrachus occipitalis (108 chants, soit 4 $\%)$, Bufo regularis (102 chants, soit 3,7 \%), Bufo maculatus (66 chants, soit 2,4\%), Amnirana albolabris (63 chants soit 2,3\%), Hyperolius "banco" et $H$. picturatus (31 chants chacun, soit $1,1 \%$ ), H. concolor (14 chants, soit $0,5 \%$ ) et Leptopelis hyloides (12 chants, soit $0,4 \%$ ).

Les coassements sont plus intenses pendant la saison humide (1511 individus ayant chanté) qu'en saison sèche (1223 individus) $\left(x^{2}=30,3\right.$; $\mathrm{ddl}=1 ; P<0,0001)$. De plus, pendant la saison pluvieuse, 327 individus ont été dénombrés le jour contre 1184 la nuit $\left(x^{2}=486,1 ; \mathrm{ddl}=1 ; P<0,0001\right)$. En saison sèche, on a enregistré 187 chants diurnes contre 1030 nocturnes $\left(x^{2}=583,9 ; \mathrm{ddl}=\right.$ $1 ; P<0,0001)$. Pour les quatre cycles, il existe une différence significative entre les abondances saisonnières.

La figure 3 présente les rythmes d'activité de chant des Anoures du complexe piscicole. Phrynobatrachus accraensis est la seule espèce diurne. Son pic d'activité de chant est atteint à 6 heures. A partir de 9 heures le nombre de mâles chantant baisse progressivement jusqu'à 15 heures. Au coucher du soleil, quelques chants isolés de mâles peuvent être enregistrés.

Les coassements de Bufo maculatus et $B$. regularis débutent à 18 heures et s'achèvent entre 3 heures et 6 heures du matin, le pic étant situé à 24 heures. Cependant, chez $B$. regularis, ces activités sont plus intenses en saison sèche qu'en saison humide. Le contraire est observé chez $B$. maculatus.
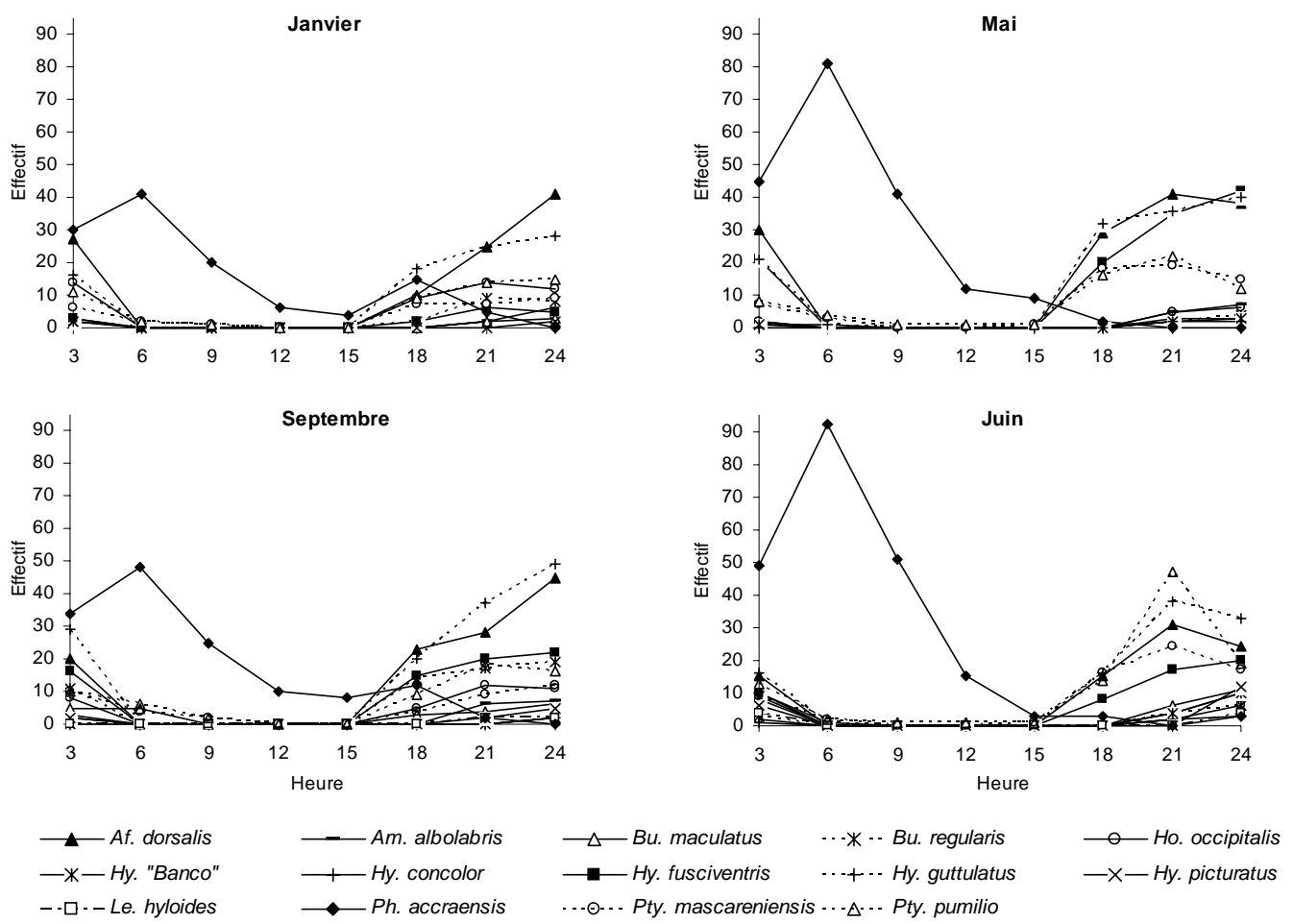

Figure 3: Variation journalière des activités de coassements des espèces d'Anoures de la ferme piscicole du Parc National du Banco pendant les saisons sèche (janvier et septembre) et pluvieuse (mai et juin) ; Hy " Banco " : Hyperoliidae nov. Gen. Nov. sp 
Chez Amnirana albolabris, Hoplobatrachus occipitalis, Ptychadena mascareniensis et Ptychadena pumilio, le pic d'activité de chant est situé entre 21 heures et 24 heures. Alors que les coassements les plus intenses débutent à 18 heures et prennent fin à 6 heures.

Les espèces de la famille des Hyperoliidae entament leurs chants à 18 heures pour les intensifier entre 21 heures et 24 heures. L'intensité des chants baisse après 24 heures pour s'arrêter totalement à de 6 heures. Dans cette famille, les chants de Afrixalus dorsalis, Hyperolius guttulatus et $H$. fusciventris sont plus abondants que ceux de $\mathrm{H}$. concolor, $\mathrm{H}$. picturatus, Leptopelis hyloides et ceux de la nouvelle grenouille arboricole de ladite famille (Hyperoliidae nov. gen. nov. sp.).

\subsection{Déterminisme des variations nycthémérales des coassements}

Les chants des grenouilles sont significativement corrélés à l'humidité relative de l'air $\left(r_{p}=0,657\right.$; ddl $=3,22 ; P<0,001)$. Les coefficients de corrélations diurnes (température : $r_{\mathrm{p}}=-0,725$; $P=0,002$; humidité relative $: r_{1}=0,814 ; P=$ 0,0001 ) sont plus élevés (en valeur absolue) que les valeurs nocturnes (température $: r_{\mathrm{p}}=-0,588$; $P=0,0165$; humidité relative $: r_{n}=0,622 ; P=$ $0,01)$. Les corrélations entre les abondances des coassements et l'intensité lumineuse ne sont pas significatives $P>0,0870$.

La figure 4 illustre l'ordination en RDA des taxons d'Anoures et les paramètres environnementaux (température, humidité relative de l'air, intensité lumineuse) qui contribuent à leur répartition nycthémérale. Les axes 1 et 2 de valeurs propres respectives $64,90 \%$ et $0,02 \%$ (soit un total de $64,92 \%)$ ont été retenus.

L'intensité lumineuse est fortement corrélée à l'axe 1. Par ailleurs, l'hygrométrie est le facteur contrôlant l'activité de coassements de la grande majorité des amphibiens de cette ferme. Elle est négativement corrélée à la température de l'air. La majorité des espèces d'amphibiens est active entre 18 heures et 3 heures du matin.

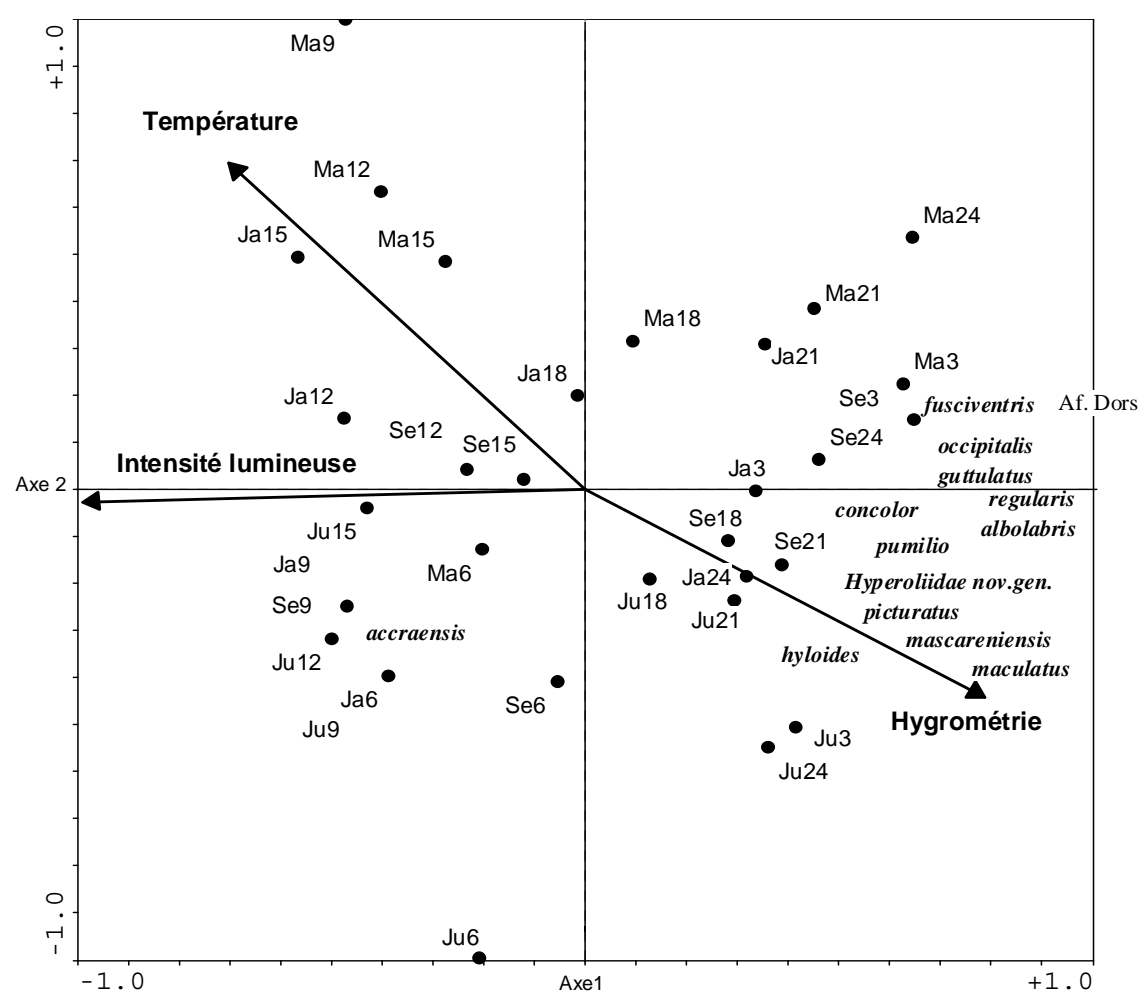

Figure 4: Ordination en RDA des taxons d'amphibiens et les paramètres environnementaux de la Ferme piscicole du Parc National du Banco. Saisons sèches $=$ janvier $(\mathrm{Ja})$ et septembre $(\mathrm{Se})$; Saisons pluvieuses $=\mathrm{mai}(\mathrm{Ma})$ et juin (Ju). Echantillons $(n=8)$ : 3-6-9-12-15-18-21-24 heures 


\section{Discussion}

Les grenouilles des familles des Bufonidae, Ranidae et Hyperoliidae du complexe piscicole du Parc National du Banco ont des activités nocturnes. A cette période de la journée, l'hygrométrie assez élevée et les températures basses seraient les facteurs conditionnant ce comportement.

Pendant la journée, la plupart se camouflent dans les formations herbacées au bord des mares ou se réfugient dans le sous-bois, les bambous et autres formations végétales entourant ce site.

La seule espèce de la famille des Petropedetidae rencontrée à la ferme piscicole (Phrynobatrachus accraensis) est très matinale. Son pic d'activité de chants est atteint à 6 heures, au moment où la température et l'intensité lumineuse sont faibles et l'humidité relative assez élevée.

Les chants isolés de quelques Bufonidae et Ranidae ont été souvent entendus après 6 heures, comme l'ont déjà observés aux Etats-Unis, Duellman \& Trueb (1986) et Rocha et al. (2000). Dans le Parc National de Taï, les grenouilles des litières du genre Phrynobatrachus sont diurnes (Rödel \& Ernest, 2002). En revanche, Cardioglossa leucomystax, Ptychadena aequiplicata et Arthroleptis sp. sont nocturnes.

L'augmentation significative de la richesse spécifique et de la densité des chants en saison pluvieuse pourrait déterminer un rythme saisonnier du comportement reproductif de ces animaux (Ovaska \& Rand, 2001). La présence permanente d'eau (16 étangs et autres retenues d'eau) expliquerait le fait qu'il n'existe pas de différences saisonnières significatives pour les abondances de grenouilles à la ferme piscicole. Les précipitations ne sont pas les seuls facteurs influençant les coassements. En effet, la température et l'humidité relative de l'air jouent aussi un rôle déterminant comme le montre nos résultats et ceux de Scott (1976) et Toft (1980). Cette influence est beaucoup plus marquée pour les intervalles de température et d'hygrométrie comprises respectivement entre $20-24^{\circ} \mathrm{C}$ et 80 - $100 \%$. En effet, ceux-ci sont propices à la reproduction, la nutrition et au développement des grenouilles (Lamotte \& Sanchez-Lamotte, 1999 ; Lampert \& Linsenmair, 2002 ; Rödel \& Ernst, 2002). Le site étant totalement ouvert, l'intensité de la lumière solaire, les fortes températures et les faibles hygrométries diurnes sont les principales causes de la chute, voire la réduction de la grande majorité des activités de chants. Lampert \& Linsenmair (2002) et Blaustein et al. (2003) ont montrés que les radiations solaires (rayons ultraviolets $\mathrm{B}$ ) sont nocives à la vie des grenouilles. Caldwell (1986) a montré que certaines espèces de grenouilles modifient leur comportement reproductif en situation de fortes variations de températures.

Les Ranidae de la ferme piscicole ont leurs pics d'activité de chants généralement situés entre 21 heures et 24 heures. Cependant, Bridges \& Dorcas (2000) ont montré que ce pic de coassements apparaît après minuit. En effet, dans notre site d'étude, l'humidité relative optimale $(85 \%)$ favorable à une activité maximale est atteinte à partir de 21 heures. Ce qui justifierait la présence d'une activité précoce chez ces grenouilles.

Amnirana albolabris chante à partir de 21 heures et s'arrête peu avant 6 heures ; le pic étant situé aux environs de 24 heures. Chez Hoplobatrachus occipitalis, Ptychadena mascareniensis et $P$. pumilio, des chants diurnes sont souvent émis par des mâles isolés en saison pluvieuse.

\section{Conclusion}

Les rythmes journaliers des activités de chants sont influencés par les variations des facteurs climatiques notamment la température, l'humidité relative. Cette communauté de grenouilles est dominée le jour par la seule espèce de la famille des Petropedetidae, à savoir, Phrynobatrachus accraensis. La nuit, les chants des Hyperoliidae, Ranidae et Bufonidae sont par ordre décroissant plus intenses. La connaissance des heures d'intenses activités de chant permettra d'élaborer une méthodologie efficiente d'inventaire systématique des Anoures. Ainsi, l'échantillonnage des espèces appartenant aux familles des Hyperoliidae, Ranidae et Bufonidae doit être réalisé entre 18 heures et 5 heures du matin. $P$. accraensis, peut être collecté de préférence de l'aurore (4 heures) à 10 heures. Cependant, pour obtenir un échantillon assez représentatif de la diversité des amphibiens d'un site donné, plusieurs techniques combinées d'échantillonnage (visuelle, acoustique, piégeage) devraient être utilisées. 


\section{Remerciements}

Ce travail fait partie d'un projet intitulé « BancoSanté-Ecologique » initié par le Laboratoire d'Environnement et de Biologie Aquatique (LEBA) de I'UFR des Sciences et Gestion de l'Environnement de l'Université d'Abobo Adjamé (Abidjan - Côte d'Ivoire).

Toute notre reconnaissance au Professeur LINSENMAIR K.E., promoteur du projet allemand «BIOTA » et aux Responsables du Parc National du Banco.

\section{Références citées}

Assemian N.E., Kouamé N.G., Tohé B., Gourène G. \& Rödel M.O., 2006. The anurans of the Banco National Parc, Côte d'Ivoire, a threatened West African rainforest. Salamandra., 42 : 41- 51.

Benito-Espinal E., 1997. La classe des batraciens, In « Flore, Faune, Monde marin ». Eds. Caraïbes. Faune $2:$ 151-159.

Bevier C.R., 1997. Utilization of energy substrates during calling activity in tropical frogs. Behav. Ecol. Sociobiol. 41: 343-352.

Biek R., Funk W.C., Maxell B.A., \& Mills L.S., 2002. What is missing in Amphibian decline research : Insights from ecological sensitivity analysis. Conserv. Biol., 16 (3) : 728-734.

Blaustein A.R., \& Kiesecker J.M., 2002. Complexity in conservation : lessons from the global decline of amphibian populations. Ecology Letters 5 : 597-608.

Blaustein A.R., Romansic J.M., Kiesecker J.M. \& Hatch A.C., 2003. Ultraviolet radiation, toxic chemicals and amphibian population declines. Diversity and Distributions 9 : 123-140.

Bridges A.S. \& Dorcas M.E., 2000. Temporal variation in Anuran calling behavior : implications for surveys and monitoring programs. Copeia $\mathrm{N}^{\circ} 2$ : 587-592.

Caldwell J.P., 1986. Selection of egg deposition site: a seasonal shift in the southern leopard frog, Rana sphecephala. Copeia : 249-253.

Channing A., 2001. Amphibian of Central and Southern Africa. Cornell University Press, Ithaca, NY.

Daget J., \& Iltis A., 1965. Poissons de Côte d'Ivoire (eaux douces et saumâtres). Mémoire de l'Institut Français d'Afrique Noire. IFAN-Dakar. $385 \mathrm{p}$.

Daszak P., Cunningham A.A., \& Hyatt A.D., 2003. Infectious diseases and amphibian populations declines. Diversity and Distributions 9 : 141-150.

Duellman W.E., \& Trueb L., 1986. Biology of Amphibians. McGraw-Hill, New York. 670p.

Given M.F., 1987. Vocalization and acoustic interactions of the carpenter frog, Rana virgatipes. Herpetologia, 43: 467-481.

Heyer W.R., Donnelly M.A., McDiarmid R.W., Hayek L.A.C., \& Froster M.S., 1994.

Measuring and monitoring biological diversity. Standard methods for amphibians. Washington \& London, Smithsonian Institution Press. 64 p.

Hilton-Taylor C., 2000. IUCN Red List of Threatened Species. IUCN Species Survival Commission, Cambridge. 21 p.

Kiesecker J.M., \& Blaustein A.R., 1995. Synergism between UV-B radiation and a pathogen magnifies amphibian embryo mortality in nature. Proceedings of the National Academy of Sciences of the USA 92 : 11049-11052.

Kiesecker J.M., \& Blaustein A.R., 1998. Effects of introduced bullfrogs and smallmouth bass on microhabitat use, growth, and survival of native red-legged frogs (Rana aurora). Conserv. Biol., 12 : 776-787.

Kiesecker J.M., Blaustein A.R., \& Belden L.K., 2001. Complex causes of amphibian population declines. Nature, 410: 681-684.

Lamotte M., \& Sanchez-Lamotte C., 1999. Adaptation aux particularités climatiques du cycle biologique d'un anoure tropical, Nectophrynoides occidentalis Angel, 1943 (Bufonidae). Alytes 16 (3-4) : 111-122.

Lampert K.P., \& Linsenmair K.E., 2002. Alternative life cycle strategies in the West African reed frog Hyperolius nitidulus : the answer to an unpredictable environment ? Oecologia 130 : 364-372.

Leistner G., 2001. Ein Vergleich der beiden Anuren Arthroleptis cf. crusculum, Angel, 1950, und Arthroleptis cf. variabilis, Matschie, 1893. Diplomarbeit, Bayerische Julius-MaximiliansUniversität Würzburg. 84 p. 
Ovaska K., \& Rand A.S., 2001. Courtship and Reproductive of the frog Eleutherodactylus diastema (Anura : Leptodactylidae) in Gamboa, Panama. J. Herpetol., 35 (1) : 44-50.

Patel T., 1993. French may eat Indonesia out of frogs. New Scientist 1868: 7 p.

Rocha C.F.D., Van Sluys, M., Alves, M.A.S., Bergallo, H.G. \& Vrcibradic D., 2000. Activity of Leaf-litter Frogs: When should Frogs be sampled? J. Herpetol., 34 (2) : 285-287.

Rödel M.-O., 2000. Herpetofauna of West Africa, Vol. I : Amphibians of the West African savanna. Edition Chimaira, Frankfurt/M., 335 p.

Rödel M.-O., \& Ernst R., 2002. A new reproductive mode for the genus Phrynobatrachus alticola has nonfeeding, nonhatching tadpoles. J. Herpetol., 36 (1) : 121-125.

Rödel M.-O., \& Ernst R., 2004. Measuring and monitoring amphibian diversity in tropical forest. I. An evaluation of methods with recommendations for standardization. Ecotropica 10: 1-14.

Runckle L.S., Wells K.D., Robb C.C., \& Lance S.L., 1994. Individual, nightly, and seasonal variation in calling behavior of the gray tree frog. Hyla versicolor: implications for energy expenditure. Behav. Ecol., 5 : 318-325.

Scott N.J., 1976. The abundance and diversity of herpetofaunas of tropical forest litter. Biotropica., $8: 41-58$.
Shimoyama R., 1989. Breeding ecology of a Japanese pond frog, Rana porosa. In: Current herpetology in East Asia.Matsui, M., Hikada, T. \& Goris, R. C. (eds.). Herpetological Society of Japan, Kyoto : 323-331.

Toft C.A., 1980. Seasonal variation in populations of Panamanian litter frogs and their prey : a comparison of wetter and drier sites. Oecologia (Berl.) 47 : 34-38.

Veith M., Kosuch J., Feltmann R., Martens H. \& Seitz A., 2000. A test for correct species declaration of frog legs imports from Indonesia into the European Union. Biodiv. and Conserv. $9: 333-341$.

Wake D.B., 1991. Declining amphibian populations. Science $253: 860$.

Wells K.D., 1977. The social behaviour of anuran amphibians. Animal Behaviour 25 : 666-693.

Williams E.S., Yuill T., Artois M., Fischer J., \& Haigh S.A., 2002. Emerging infectious diseases in wildlife. Rev. Sci. Tech. Off. Int. Epizooties., 21 (1) : 139-157.

Zimmerman B.L., 1994. Audio strip transects. In Heyer, W.R., Donnelly, M.A., McDiarmid R.W., Hayek, L.-A.C., et Froster, M.S., (eds.) : Measuring and monitoring biological diversity. Standard methods for amphibians. Washington \& London, Smithsonian Institution Press : 92-97. 